\title{
EFFECTS OF DEPOSITION CONDITIONS ON TEXTURE IN COPPER THIN FILMS ON Si (111)
}

\author{
D. B. KNORR $\dagger$ and T.-M. LU \\ Center for Integrated Electronics, Rensselaer Polytechnic Institute, \\ Troy, NY 12180-3590, USA
}

\begin{abstract}
The texture of copper films deposited on bare $\mathrm{Si}(111)$ is studied using a partially ionized beam deposition technique. A change in deposition parameters (percent ions and substrate bias) radically alters the texture from epitaxial to fiber. Under conditions indicative of deposition by evaporation, a sharp texture typified by a $\mathrm{Cu}\{531\} \| \mathrm{Si}(111)$ relationship is observed. At measurable ion energy levels, the film texture becomes $\mathrm{Cu}(111)$. A narrowly defined set of conditions promotes epitaxy which breaks down into a fiber texture for conditions of successively higher ion energy. The role of internal (boundary) energy and interfacial (substrate/film and film/ambient) energy are discussed.
\end{abstract}

KEY WORDS Thin film, copper, ion beam deposition, texture, epitaxy, fiber texture.

\section{INTRODUCTION}

Characterization of microstructure in thin metallic films is important for understanding the effects of deposition conditions on properties of the film. Diverse applications such as decorative coatings, corrosion protection, optical coatings, wear resistance, and microelectronics are represented. Much work has focused on the microstructure of the films as defined by defect content and by the grain morphology as represented by the zone model of Movchan and Demchishin (1969) with modifications by Thornton (1977). Substantially less effort has been expended to understand, or even simply to characterize, the crystallographic texture in metal films. This stands in sharp contrast to bulk materials where development of texture and microstructure during thermomechanical processing has been thoroughly documented. Further work on bulk systems serves to supplement and refine existing studies, to obtain a fundamental understanding of the operative deformation, recrystallization, and grain growth mechanisms, and to provide input to mechanical behavior models.

Few studies have addressed texture in thin metallic films although strong textures usually develop as a consequence of film deposition. Texture characterization usually consists of a comparison of peak heights from an $\theta / 2 \theta$ diffraction scan from the thin film (e.g. Yapsir, You, and Lu, 1989). When texture components are low index planes, a texture index such as $I_{(111)} / I_{(200)}$ is

$\dagger$ Also Materials Engineering Department.

$\ddagger$ Also Physics Department. 
used. Examples are studies on electromigration in aluminum alloy films (Vaidya and Sinha, 1981) and on partially ionized beam deposition of copper (Bai et al., 1990). Pole figure analysis is rarely used, yet it provides information on the character, strength, and sharpness of the texture (Knorr and Lu, 1989). ODF analysis of textures is virtually unknown in the study of thin film microstructure, especially as applied to systems used in microelectronic applications.

Thin film textures vary between two extremes. Epitaxial films are essentially single crystal in character and are deposited on bulk single crystal substrates. Preferred lattice matching between the deposited material and the substrate develops specific crystallographic relationships (Zur and McGill, 1984). Grain boundaries are not present although twin boundaries are observed in systems where specific twin relationships exist. Another extreme is represented by a fiber texture which develops most often when films are deposited on amorphous substrates. A specific crystallographic orientation is observed where the closest packed atomic plane is parallel to the surface of the substrate. In face centered cubic systems an (111) plane is parallel to the surface where a rotation of infinite order about the (111) pole defines the fiber character of the texture. Such textures have been characterized for gold on glass (Witt, Vook, and Schwartz, 1965) and for the aluminum on $\mathrm{SiO}_{2}$ (Knorr and Lu, 1989). Polycrystalline textures on single crystal substrates are often sharper than textures on amorphous substrates due to the strong influence of the underlying single crystal lattice. If polycrystalline films form, a strong substrate influence is often retained.

A variety of vacuum deposition techniques are available for deposition of thin metallic films. They fall into two major classes: chemical vapor deposition (CVD) and physical vapor deposition (PVD). PVD techniques are subdivided further into technologies such as sputtering, evaporation, and ion assisted deposition using either foreign ions or self-ions. This report will consider an ion-assisted deposition technique called Partially Ionized Beam (PIB) (Yapsir, You, and Lu, 1989).

A controlled percentage of self-ions is generated, then accelerated to the substrate by a bias potential on the target. Documented advantages of the technique for metal deposition include an equiaxed rather than columnar microstructure for nominally room temperature deposition (Selvaraj et al., 1987), excellent in-situ cleaning (Yapsir, Lu, and Lanford, 1988), excellent adhesion of metal, smooth film surfaces and reduced spike formation at the $\mathrm{Al} / \mathrm{Si}$ interface for deposition in a conventional vacuum at room temperature (Choi et al., 1988). Conventional vacuum conditions $\left(10^{-4} \mathrm{~Pa}\right)$ are used rather than the ultra high vacuum environments characteristic of other deposition techniques such as molecular beam epitaxy.

\section{EXPERIMENTAL}

Figure 1 is a schematic diagram of the PIB deposition geometry. High purity copper is vaporized in a graphite crucible about $1 \mathrm{~cm}$ in diameter using electron bombardment heating of the crucible. The evaporated copper vapor is partially ionized by electron impact above the mouth of the crucible. The electrons for bombardment and for impact ionization are provided by the same filament. A 


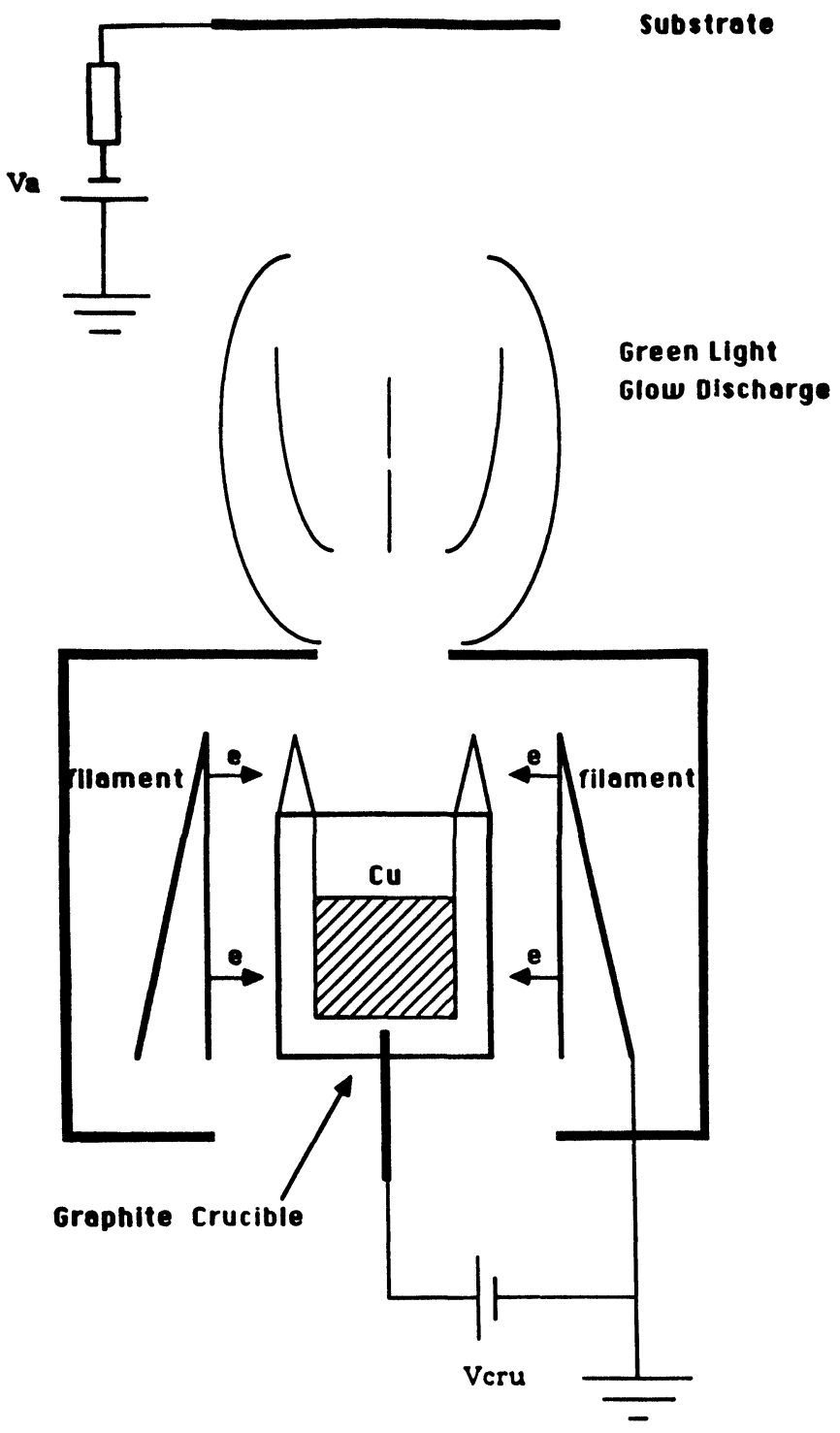

Figure 1 Schematic representation of the partially ionized beam deposition apparatus.

bias voltage, $V_{b}$, on the silicon substrate accelerates the ions during deposition. The ion percentage in the beam is determined by measuring the ratio of the total ion current received at the substrate and the deposition rate. A wide variety of deposition conditions are established by varying the bias voltage between $0 \mathrm{kV}$ and $5 \mathrm{kV}$ and the copper ion content between 0 and $8 \%$. Ion fraction and bias voltage are maintained at levels below which either ion implanation or substrate sputtering occur. 
Table 1 Partially ionized beam deposition conditions

\begin{tabular}{lllll}
\hline Condition & $\begin{array}{l}\text { Bias voltage, } V_{b} \\
(k V)\end{array}$ & \% ions & $\begin{array}{l}\text { Deposition rate } \\
(A / \mathrm{sec})\end{array}$ & $\begin{array}{l}\text { Film } \\
\text { thickness }(\mathrm{nm})\end{array}$ \\
\hline 1 & $\sim 0$ & $\sim 0$ & 10.5 & 200 \\
2 & 1.0 & 2.1 & 14 & 150 \\
3 & 1.4 & 2.6 & 17 & 300 \\
4 & 5.3 & 1.8 & 4 & 200 \\
\hline
\end{tabular}

The substrates were quarter sections of $7.62 \mathrm{~cm}$ diameter $n$-type $\mathrm{Si}$ (111) wafers with a resistivity of $4.25-5.25 \Omega-\mathrm{cm}$. All wafers were chemically cleaned by the RCA process (Kern and Puotlineu, 1970). No in-situ cleaning was performed prior to deposition. Deposition was done nominally at room temperature, but bulk substrate temperature rises to about $50^{\circ} \mathrm{C}$ during deposition due to source radiation. The base vacuum is $3 \times 10^{-5} \mathrm{~Pa}$ while the vacuum during deposition decreases to $1 \times 10^{-4} \mathrm{~Pa}$. Deposition conditions are given in Table 1 .

The copper film was evaluated by two X-ray diffraction technique to determine the orientation between the film and the Si (111) substrate. An $\theta / 2 \theta$ scan located the major reflections in the copper film. Pole figure analysis determined the intensity distribution of $\mathrm{Cu}(111)$ poles by the Schultz (1949) reflection technique to a tilt angle of $80^{\circ}$ or $85^{\circ}$ from the sample normal direction. Raw data were corrected for geometrical defocusing and for absorption since the film thickness is less than the X-ray penetration depth. The orientation of the single crystal silicon was obtained by fixing the diffraction condition on $\mathrm{Si}(111)$ and scanning at a tilt angle of $70.5^{\circ}$ to locate the three $\{111\}$ orientations that complement the (111) whose pole is normal to the specimen surface. $\mathrm{Cu}(111)$ pole figures are plotted with intensity contours normalized such that random intensity is represented by a value of one. The orientations of the four $\mathrm{Si}(111)$ poles are also included to define the substrate orientation.

\section{RESULTS}

Four conditions of copper on bare $\mathrm{Si}(111)$ were deposited. The deposition conditions are summarized in Table 1 where particular note should be taken of the bias potential and percent ion. Radically different textures result from the varied deposition environments. Condition 1 (Knorr, Bai, and $\mathrm{Lu}, 1990$ ) and condition 2 (Bai et al., 1990) were discussed in their respective references and will only be summarized here. Condition 3 and condition 4 are uniquely different from either condition 1 or condition 2.

For condition 1, no potential is deliberately applied to the substrate, but the potential is allowed to "float" resulting in a small $(<0.5 \mathrm{kV})$ bias. The $\mathrm{Cu}(111)$ pole figure is shown in Figure 2 which presents a very strong and unusual texture. Taking the seven strong intensity regions as ideal orientations, texture analysis shows that $\mathrm{Cu}\{531\}$ is parallel to $\mathrm{Si}(111)$. In the plane of the film, two variants of copper orientation are present with orientation relationships $\mathrm{Si}\langle 11 \overline{2}\rangle \| \mathrm{Cu}\langle 1 \overline{3} 4\rangle$ and $\mathrm{Si}\langle 11 \overline{2}\rangle \| 2.5^{\circ}$ from $\mathrm{Cu}\langle 3 \overline{5} 0\rangle$. Equivalent intensity 


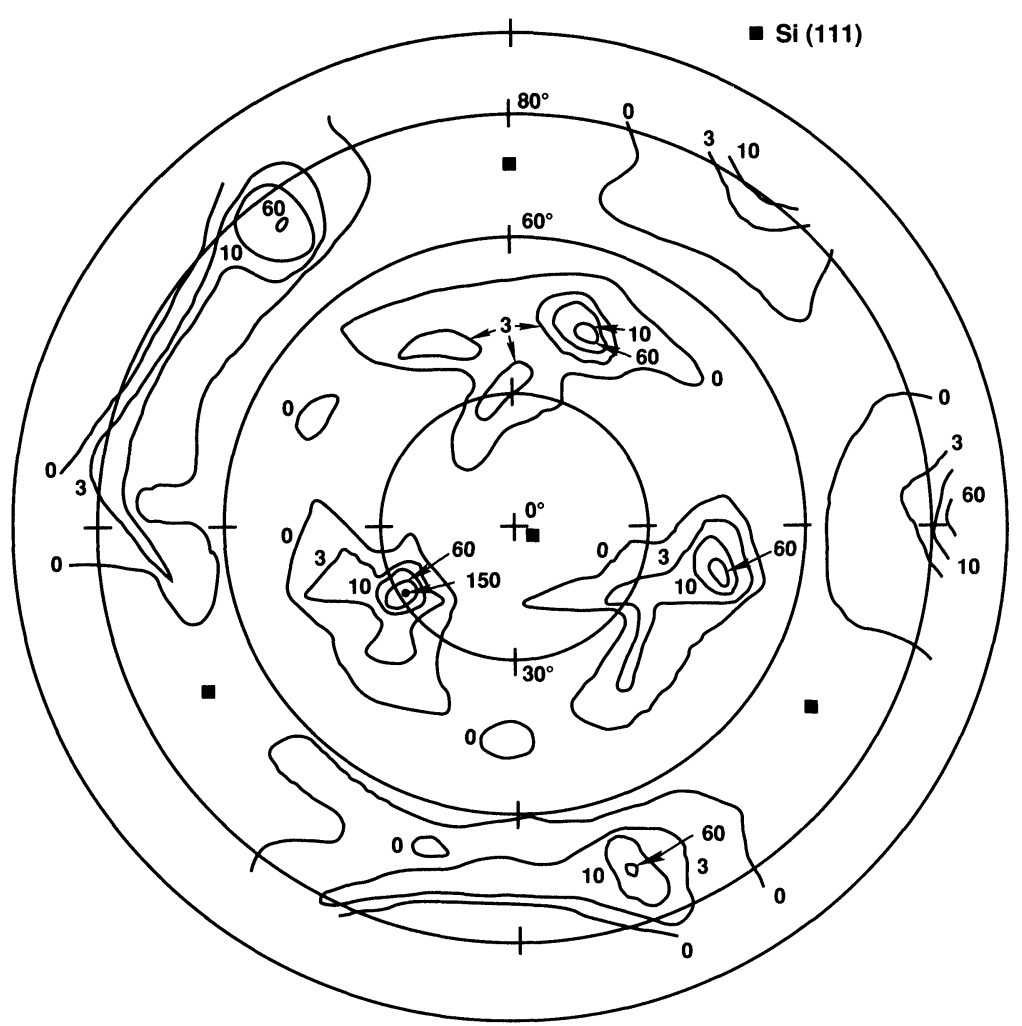

Figure $2 \mathrm{Cu}$ (111) pole figure for condition 1. The orientation of the $\mathrm{Si}(11)$ substrate is indicated.

values indicate that an equal volume fraction of each variant is present. A rotation of $56.5^{\circ}$ about the maximum intensity point in Figure 2 is required to rotate one variant into the other. This is less than the $60^{\circ}$ rotation for $\mathrm{Cu}$ (111) twin variants but somewhat greater than the $55.1^{\circ}$ between $\langle 13 \overline{4}\rangle$ and $\langle\overline{3} 05\rangle$ which are weakly epitaxial relationships.

The condition 2 copper film is the only one to achieve epitaxy on an $\mathrm{Si}$ (111) substrate. The orientation relationship is shown in Figure 3 . The epitaxial relationship given $\mathrm{Cu}(111) \| \mathrm{Si}(111)$ is $\mathrm{Cu}\langle\overline{1} 10\rangle \| \mathrm{Si}\langle\overline{1} 10\rangle$. The second copper orientation variant is the twin orientation of the first which involves a rotation of $60^{\circ}$. Further analysis confirms that the epitaxy occurs through a very thin interfacial layer of $\eta-\mathrm{Cu}_{2} \mathrm{Si}$.

Condition 3 represents a breakdown of the epitaxial relationship shown for condition 2. Figure 4 gives the $\mathrm{Cu}(111)$ pole figure with $\mathrm{Si}(111)$ substrate orientation as indicated. Two different texture components are present. Strong intensity locations indicate some near-epitaxial relationship while the remaining material forms a fiber texture. Integration of the intensity profile shows that $48 \%$ of the grains belong to the near epitaxial component while $52 \%$ of the grains distribute themselves as a fiber texture component. An important contrast with 


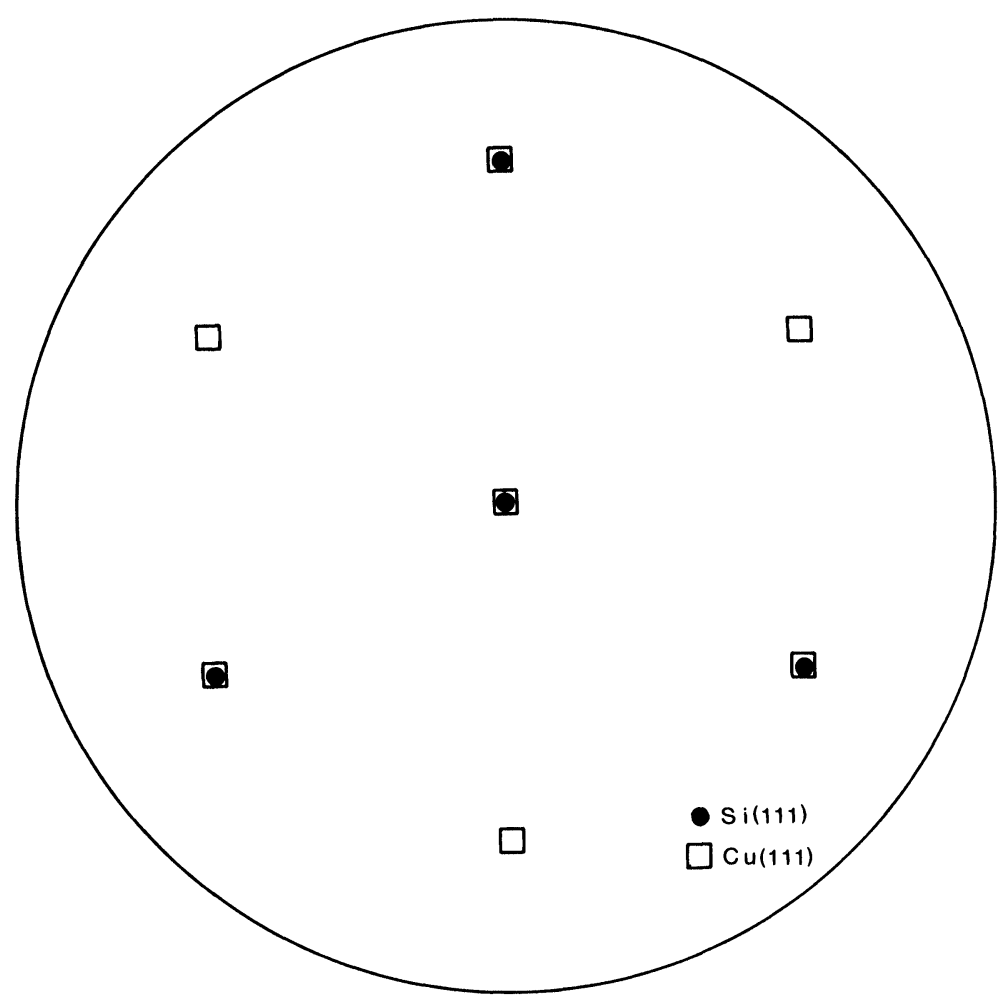

Figure 3 Pole figure for condition 2 showing the epitaxial relationship between copper and silicon.

the expitaxial texture in Figure 3 concerns the $\mathrm{Si}(111)$ orientation which is rotated approximately $15^{\circ}$ from the orientation shown in Figure 3.

Condition 4 is a pure $\mathrm{Cu}(111)$ fiber texture. Figure 5 shows the pole intensity versus tilt angle which fully describes the texture. A broad (111) distribution is evident, but no other texture component (e.g. (200) fiber) is found. A much tighter spread of poles is seen for condition 3 as compared to condition 4 in Figure 5.

\section{DISCUSSION}

Deposition conditions of the PIB film are clearly responsible for substantial change in the film orientation. The ion content and substrate bias control the ion energy for deposition done otherwise at room temperature. Epitaxy is observed over a narrowly defined set of conditions. The important factors that promote epitaxy are known to be surface cleanliness and atom mobility. Ion assisted deposition cleans the substrate surface by energetic ion bombardment which sputters and thermally desorbs (what is presumed to be) monolayers of absorbate. A conventional vacuum is sufficient because in-situ cleaning during 


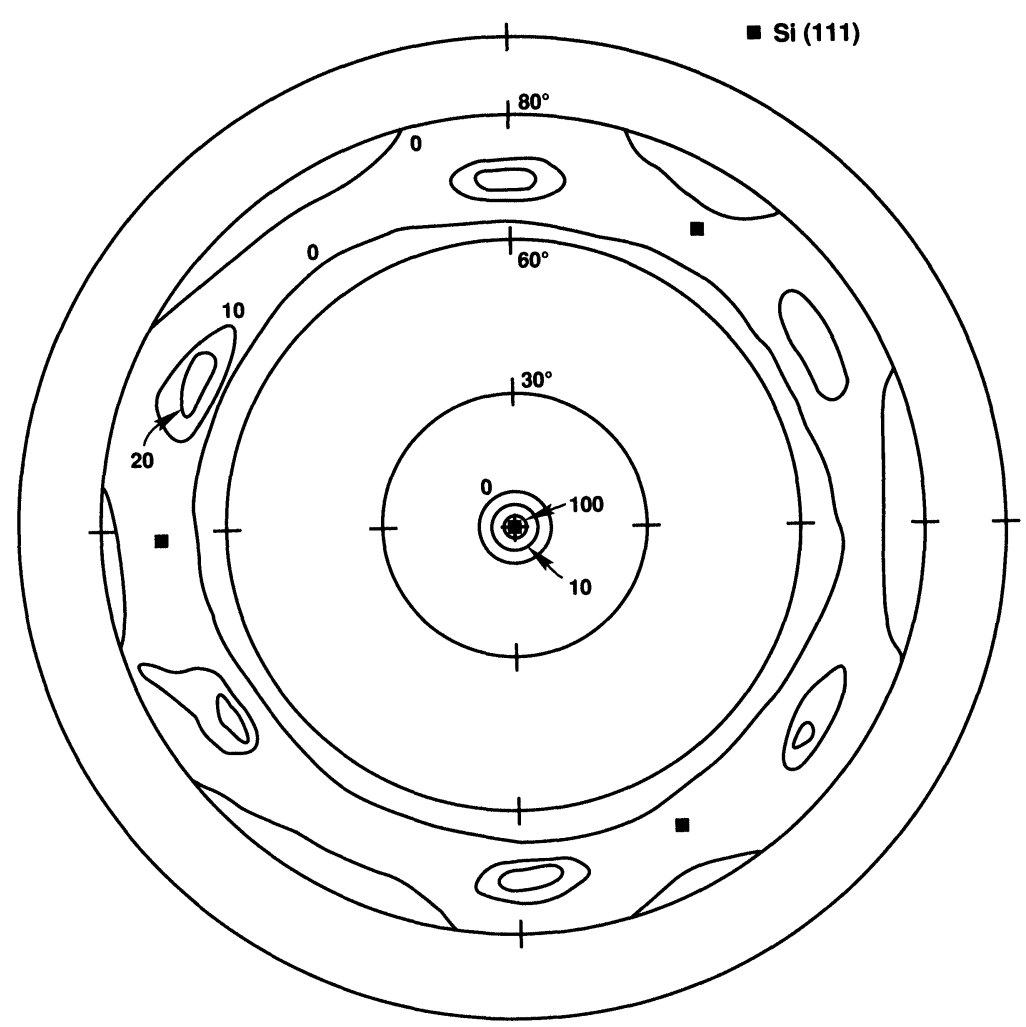

Figure $4 \mathrm{Cu}$ (111) pole figure for condition 3. The orientation of $\mathrm{Si}(111)$ substrate is indicated.

depositions maintains a clean interface. When very little bias or ion content is used (condition 1), in-situ cleaning is less efficient. The resulting near epitaxial conditions is not the low energy $\mathrm{Cu}(111)$ but an unexpected $\mathrm{Cu}\{531\}$ which is only weakly epitaxial based on poor lattice matching with the $\mathrm{Si}(111)$ substrate. It is important to note, however, that lattice matching is better for $\mathrm{Cu}\{531\}$ than for random orientations of atomic lattices across the film/substrate interface.

The second element impacting epitaxy is atomic surface mobility. Ion assisted deposition uses ions to deposit energy onto the substrate. Collisions transfer energy without bulk heating of the substrate which increases surface mobility. A window in atom energy appears to exist where too low energy restricts atom surface mobility while high bias conditions such as condition 4 might locally deposit its energy without optimal transfer to surface atoms.

Condition 3 represents a transition between epitaxy and a fiber texture. Approximately equal fractions are present. It is important to note that the lattice matching present in condition 2 is not observed in the epitaxial component is condition 3 where a rotation of $15^{\circ}$ is present. Although no microstructural evaluation was done for this condition, scanning electronmicroscopy of a cross-section from a similar sample $(2.5 \mathrm{kV}$ bias and $4.8 \%$ ions) showed a duplex 


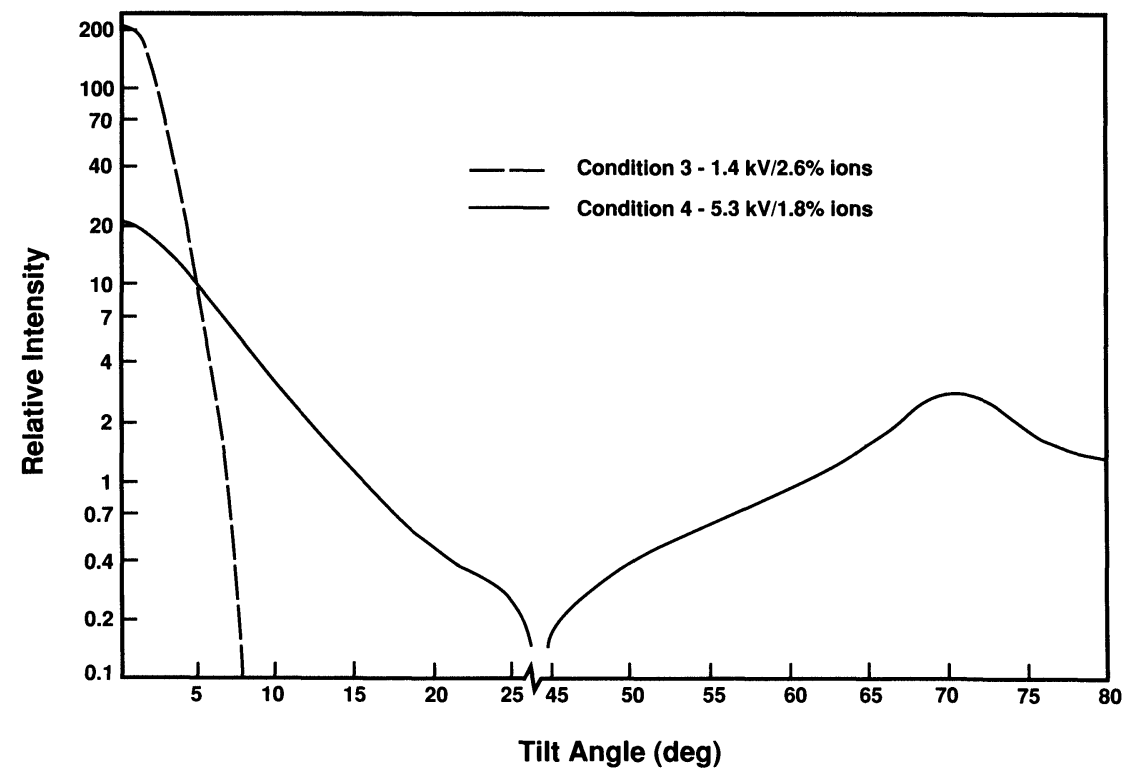

Figure $5 \mathrm{Cu}$ (111) pole intensity versus tilt angle for the fiber textured condition 4 . The near normal $\mathrm{Cu}$ (111) pole distribution for condition 3 is shown for comparison.

microstructure. A columnar grain morphology was present through approximately $40 \%$ of the film cross-section while the remaining thickness was equiaxed. This result implies that the duplex texture in the condition 3 film is a consequence of a duplex microstructure. Columnar grains strongly influenced by the substrate could be responsible for the near epitaxial component and equiaxed grains for the fiber component.

Three energy contributions determine the film orientation. Two interfacial energy components are substrate/film and film/ambient ("vacuum"). The most important internal energy term is boundary energy tied to grain boundaries and to twin boundaries. If surface mobility is sufficiently high (bias and ions present), a $\mathrm{Cu}$ (111) film will result because close packed planes are known to grow preferentially. Minimizing the substrate/film interfacial energy by lattice matching promotes epitaxy. Internal energy contributes to the character of the $\mathrm{Cu}(111)$ texture. Nucleation, growth, and coarsening of the film microstructure contributes by determining how boundary energy is minimized. In order of decreasing energy, boundary energies can be ranked as high angle, low angle, and twin. Epitaxial films have only twin boundaries. Very sharp but polycrystalline textures such as condition 3 will contain both twin and low angle grain boundaries. Finally, a fiber texture will have a substantial fraction of high angle grain boundaries because of random orientations between grains rotated about their (111) axis.

The only comparable work is by Namba and Mori (1976) who deposited $300 \mathrm{~nm}$ silver film on single crystal $\mathrm{NaCl}$. Ionization of $10 \%$ of the silver atoms and bias of $3 \mathrm{kV}$ produced an $\mathrm{Ag}(200)$ epitaxial condition while evaporation produced a polycrystalline film. No texture analysis was done on the evaporated silver film, 
but the transmission electron microscopy results indicate a texture with a strong $\mathrm{Ag}$ (111) character, possibly fiber. Namba and Mori (1976) interpret their results in terms of the role of defect creation on epitaxy during ion bombardment and in terms of an "entropy decrease" driving ion bombardment without specifying a mechanism. Bunshaw (1982) discusses these same results in terms of increased surface mobility of the ionized specie where he takes the effective surface temperature to be higher during ion bombardment.

The concept of effective surface temperature is helpful in explaining the equiaxed microstructure of PIB films. The zone models of Movchan and Denchishin (1969) and of Thornton (1977) do not predict an equiaxed microstructure until $T \geqq 0.5 T_{m}$ in pure metals. PIB copper films are equiaxed (Zone 3) after deposition at bulk temperatures $\sim 0.3 T_{m}$ where a columnar Zone $T$ or Zone 2 structure might normally be expected. The energy deposited locally by ion bombardment raises the effective temperature of the near surface layer of a film which promotes the equiaxed film growth. Likewise, bias sputtering of copper onto copper at room temperature also produces an equiaxed microstructure (Patten, McClanahan, and Johnson, 1971). No analog to the zone model has yet been proposed for ion assisted deposition where normalized temperature would be replaced by a parameter that reflects the energy in an atom ( + ion) beam.

\section{CONCLUSIONS}

1. Deposition of copper films by a partially ionized beam produces a surprising variety of textures depending on the deposition conditions.

2. High deposition energy promotes the formation of a $\mathrm{Cu}(111)$ fiber texture despite the strong influence of a single crystal $\mathrm{Si}(111)$ substrate.

3. A narrow processing window exists to develop an epitaxial film of $\mathrm{Cu}(111) \| \mathrm{Si}(111)$.

4. Interfacial and internal energy terms contribute to the texture that is observed. $\mathrm{Cu}(111)$ is favored when even minimal surface mobility is imparted by ion deposition.

\section{References}

Bai, P., Yang, G.-R., Lu, T.-M., and Lau, L. W. M. (1990). Deposition of Cu Film on $\mathrm{SiO}_{2}$ using a Partially Ionized Beam. Journal of Vacuum Science and Technology, A8,1465-1469.

Bai, P., Yang, G.-R., You L., Lu, T.-M., and Knorr, D. B. (1990). Room-Temperature Epitaxy of $\mathrm{Cu}$ on Si (111) Using Partially Ionized Beam Deposition. Journal of Materials Research, 5, 989-997.

Bunshah, R. F. (1982). Evaporation. in Deposition Technologies for Films and Coatings, edited by R. F. Bunshaw, pp. 83-169. Park Ridge, New Jersey: Noyes Publications.

Choi, C.-H., Harper, R. A., Yapsir, A. S., and Lu, T.-M. (1988). Epitaxial Growth of $\mathrm{Al}(111) / \mathrm{Si}$ (111) Films Using Partially Ionized Beam Deposition. Applied Physics Letters, 51, 1992-1994.

Kern, W. and Puotlineu (1970). Cleaning Solutions Based on Hydrogen Peroxide for Use in Silicon Semiconductor Technology. RCA Review, 31, 187-206.

Knorr, D. B., Bai, P., and Lu, T.-M. (1990). An Unusual Orientation Relationship for a Copper Film on Si (111). Applied Physics Letters, 56, 1859-1861. 
Knorr, D. B. and Lu, T.-M. (1989). Texture Analysis of $\mathrm{Al} / \mathrm{SiO}_{2}$ Films Deposited by a Partially Ionized Beam. Applied Physics Letters, 54, 2210-2212.

Movchan, B. A. and Demchishin, A. V. (1969). Study of the Structure and Properties of Thick Vacuum Condensates of Nickel, Titanium, Tungsten, Aluminum Oxide and Zirconium Dioxide. Physical Metallurgy and Metallography, 28, 83-90.

Namba, Y. and Mori, T. (1976). Epitaxial growth of Ag Deposited by Ion Deposition on NaCl. Journal of Vacuum Science and Technology, 13, 693-697.

Patten, J. W., McClanahan, E. D., and Johnson, J. W. (1971). Room-Temperature Recrystallization in Thick Bias-Sputtered Copper Deposits. Journal of Applied Physics, 42, 4371-4377.

Schultz, L. G. (1949). A Direct Method of Determining Preferred Orientation of a Flat Reflection Sample Using a Geiger Counter X-Ray Spectrometer. Journal of Applied Physics, 20, 1030-1036.

Selvaraj, R., Yang, S.-N., MacDonald, J. F., and Lu, T.-M. (1987). Ion Cluster Beam Metallized Interconnections for Water Scale Integration. In Proceedings IEEE VLSI Multilevel Interconnection Conference, edited by T. E. Wade, pp. 440-448. New York: Electron Device Society.

Thornton, J. A. (1977). High Rate Thick Film Growth. Annual Review of Materials Science, 7, 236-260.

Vaidya, S. and Sinha, A. K. (1981). Effect of Texture and Grain Structure on Electromigration in $\mathrm{Al}-0.5 \% \mathrm{Cu}$ Thin Films. Thin Solid Films, 75, 253-259.

Witt, F., Vook, R. W., and Schwartz, M. (1965). Quantitative Texture Measurements on Evaporated Films. Journal of Applied Physics, 36, 3686-3687.

Yapsir, A. S., Bai, P., and Lu, T.-M. (1988). Formation of Low Temperature Al/n-Si Schottky Contacts Using Partially Ionized Beam Deposition Techniques. Applied Physics Letters, 53, 905-907.

Yapsir, A. S., Lu, T.-M. and Lanford, W. A. (1988). Reduction of Interface Hydrogen Content by Partially Ionized Beam Deposition Technique. Applied Physics Letters, 51, 1962-1964.

Yapsir, A. S., You, L., and Lu, T.-M. (1989). Partially Ionized Beam Deposition of Oriented Films. Journal of Materials Research, 4, 343-349.

Zur, A. and McGill, T. C. (1984). Lattice Match: An Application to Heteroepitaxy. Journal of Applied Physics, 55, 378-386. 